Impacto de la homologación del IVA en el consumo de los hogares de Baja California, Baja California Sur y Quintana Roo, México

Rolando Israel Valdez Ramírez y Emilio Hernández Gómez
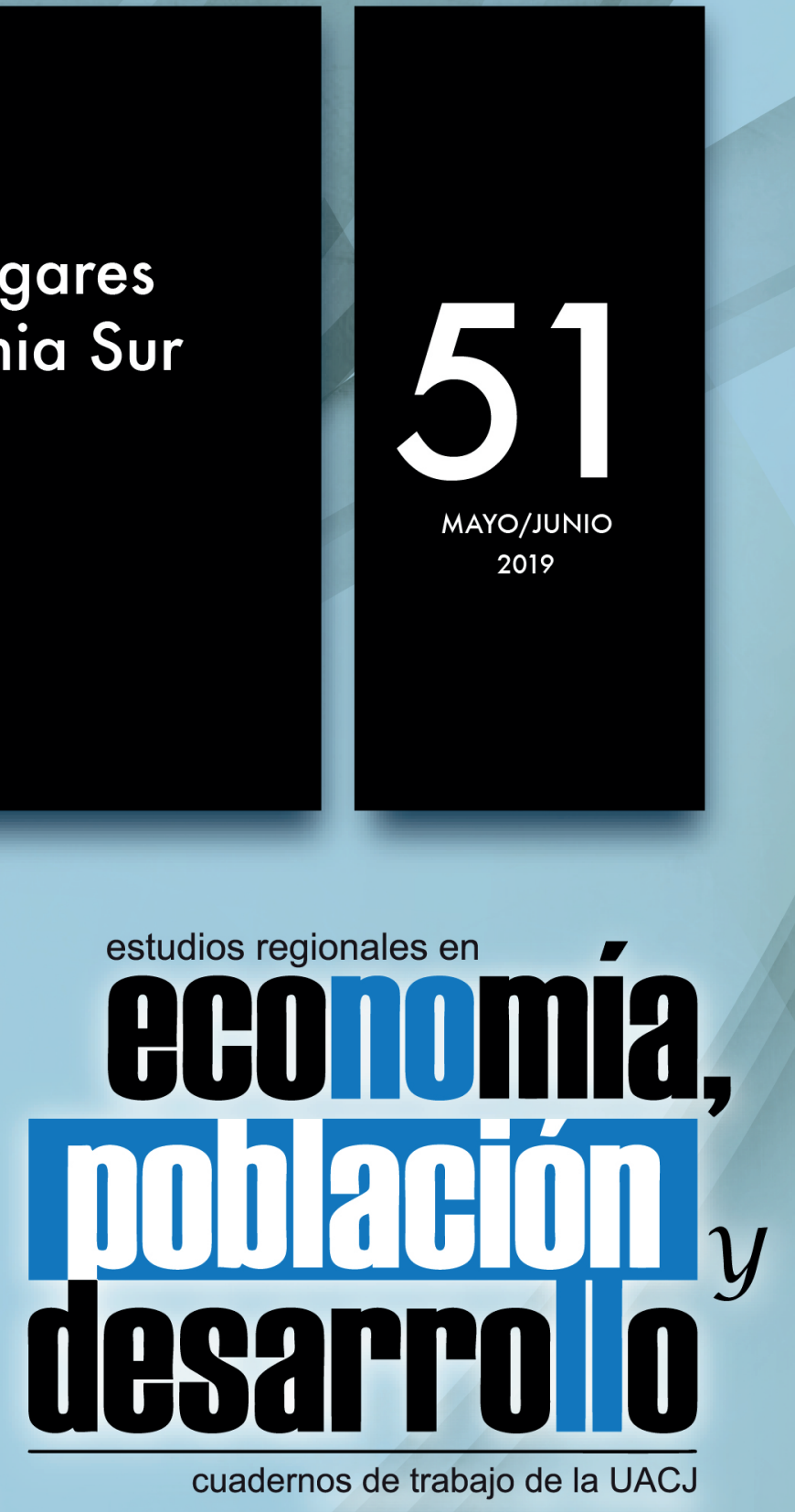


\title{
Impacto de la homologación del IVA en el consumo de los hogares de Baja California, Baja California Sur y Quintana Roo, México
}

\author{
Rolando Israel Valdez Ramírez y Emilio Hernández Gómez
}

UNIVERSIDAD AUTÓNOMA DE CIUDAD JUÁREZ RED IBEROAMERICANA DE ESTUDIOS DEL DESARROLLO 


\title{
UNIVERSIDAD AUTÓNOMA DE CIUDAD JUÁREZ RED IBEROAMERICANA DE ESTUDIOS DEL DESARROLLO
}

\author{
Cuerpo Académico de Estudios Regionales en \\ Economía, Población y Desarrollo
}

Universidad Autónoma de Ciudad Juárez

2018-2024

Mtro. Juan Ignacio Camargo Nassar

Rector

Mtro. Daniel Alberto Constandse Cortez

Secretario General

Mtro. Jesús Meza Vega

Director General de Comunicación Universitaria

Dra. Beatriz Araceli Díaz Torres

Coordinadora General de Investigación y Posgrado

Comité de Coordinación de la Red Iberoamericana de Estudios del Desarrollo 2018-2020

Dra. Paulina Sanhueza Martínez (Universidad de la Frontera, Chile)

Coordinadora General

Dr. Ignacio Rodríguez Rodríguez (Universidad de la Frontera, Chile)

Secretario general

Dra. Myrna Limas Hernández

(Universidad Autónoma de Ciudad Juárez, México)

Vocal de Organización

Dr. Pablo Galaso Reca (Universidad de la República, Uruguay)

Vocal de Organización

Dr. Luis Enrique Gutiérrez Casas

Director y editor de Cuadernos de Trabajo

Estudios Regionales en Economía, Población y Desarrollo

Comité editorial

Sección internacional Dra. Sofía Boza Martínez

(Universidad de Chile, Chile)

Dra. Olga Biosca Artiñano

(Glasgow Caledonian University, Reino Unido)

Dra. Ángeles Sánchez Díez

(Universidad Autónoma de Madrid, España)

Dr. Thomas Fullerton Mankin

(University of Texas at E1 Paso, Estados Unidos)

Dr. Adrián Rodríguez Miranda

(Universidad de la República, Uruguay)

Dra. Ikuho Kochi

(Kanazawa University, Japón)

Sección local

(Universidad Autónoma de Ciudad Juárez)

Dra. Myrna Limas Hernández

Dra. Rosa María García Almada

Dr. Raúl Alberto Ponce Rodríguez

Dr. Isaac Leobardo Sánchez Juárez

Dr. Héctor Alonso Barajas Bustillos

Dr. Juan Carlos Medina Guirado

Diseño de cubierta Abigail Bautista
Estudios Regionales en Economía, Población

y Desarrollo. Cuadernos de Trabajo de la UACJ

ISSN 2007-3739

Número 51. Mayo/Junio 2019

Impacto de la homologación del IVA en el consumo

de los hogares de Baja California, Baja California Sur y

Quintana Roo, México

Rolando Israel Valdez Ramírez y Emilio Hernández Gómez

Universidad Autónoma de Ciudad Juárez

Estudios Regionales en Economía, Población y Desarrollo, Cuadernos de Trabajo de la UACJ

Año 9, No. 51 mayo - junio 2019, es una publicación bimestral editada por la Universidad Autónoma de Ciudad Juárez a través del Cuerpo Académico de Estudios Regionales en Economía, Población y Desarrollo del Instituto de Ciencias Sociales y Administración. Redacción: Avenida Universidad y H. Colegio Militar, Zona Chamizal s/n., C.P. 32300, Ciudad Juárez, Chihuahua, México. Teléfonos: (656) 688-38-00, ext. 3792. Correo electrónico: igtz@uacj.mx.

Editor responsable: Luis Enrique Gutiérrez Casas. ISSN 2007-3739, Impresa por Studio Los Dorados, calle Del Campanario, número 820-2, Santa Cecilia, C.P.32350, Cd. Juárez, Chihuahua. Distribuidor: Subdirección de Gestión de Proyecto y Marketing Editorial. Ave. Plutarco Elías Calles 1210, Foviste Chamizal, C.P. 32310, Ciudad Juárez, Chihuahua.

Este número se terminó de imprimir el 15 de abril, 2019 con un tiraje de 120 ejempares.

Los ensayos publicitarios son responsabilidad exclusiva de sus autores.

Se autoriza la reproducción total o parcial bajo condición de citar la fuente.

Registrada en: EBSCO RePEC

Publicación afiliada a la Red Iberoamericana de Estudios del Desarrollo

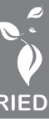

Universidad Autónoma de Ciudad Juárez

Ave Plutarco Elías Calles 1210

Foviste Chamizal, C.P. 32310

Ciudad Juárez, Chihuahua, México

www.uacj.mx

(C) Universidad Autónoma de Ciudad Juárez 


\title{
Impacto de la homologación del IVA en el consumo de los hogares de Baja California, Baja California Sur y Quintana Roo, México
}

\author{
Rolando Israel Valdez Ramírez * y Emilio Hernández Gómez **
}

\begin{abstract}
Resumen
En este estudio es evaluado el impacto de la reforma a la ley del IVA de 2013 en el consumo de los hogares fronterizos, el caso de los estados mexicanos de Baja California, Baja California Sur y Quintana Roo. Para hacer esta evaluación es utilizada la metodología de diferencia en diferencias a través de modelos Tobit sobre 25 bienes que componen el consumo de los hogares. Las estimaciones son llevadas a cabo con datos de la Encuesta Nacional de Ingreso y Gastos de los Hogares (ENIGH) 2012 y 2014. Los resultados revelan un impacto negativo de $11.4 \%$ sobre el consumo de los hogares una vez entrada en vigor la nueva ley del IVA, a su vez, los tres componentes del consumo más afectados con esta reforma son: combustible, mantenimiento de vehiculo y comunicaciones.
\end{abstract}

Palabras clave: Evaluación de impacto, impuesto al valor agregado, consumo, México.

\begin{abstract}
In this essay, I analyze the impact of the increase in the Value Added Tax (IVA) in 2013 on household consumption in the Mexican Border States of Baja California, Baja California Sur and Quintana Roo. The estimations consider a difference in differences methodology with a Tobit model for 25 individual goods. Data come from the National Survey of Households'Income and Spending (ENIGH) for 2012 and 2014. The results show a decrease of $11.4 \%$ on household consumption after the tax increase. The negative impact is particularly large in fuels, automotive maintenance. and communications.
\end{abstract}

Keywords: Impact assessing, value added tax, consumption, Mexico.

JEL Classification: H20, H30, R10.

- Recibido en: Agosto de 2018.

- Aprobado en: Enero 2019.

* Profesor-Investigador de la Facultad de Economía y Relaciones Internacionales de la Universidad Autónoma de Baja California. Correo electrónico: rvaldez35@uabc.edu.mx.

** Profesor-Investigador de la Facultad de Economía y Relaciones Internacionales de la Universidad Autónoma de Baja California. Correo electrónico: emiliohernandez@uabc.edu.mx. 


\section{○ 1. Introducción.}

El objetivo del presente estudio es evaluar el impacto de la reforma a la ley del Impuesto al Valor Agregado (IVA) llevada a cabo en 2013 y que entró en vigor a partir del 01 de enero de 2014, la cual, entre otros aspectos, incluía elevar la tasa de $11 \%$ a $16 \%$ en la región fronteriza del país, homologando de esta manera el impuesto que es pagado en el resto del país. Esta evaluación es llevada a cabo a través de la técnica denominada Diferencia en Diferencias (DD), misma que se encuentra dentro de las técnicas cuasi experimentales, debido a que es definido un grupo de tratamiento (Baja California, Baja California Sur y Quintana Roo), así como un grupo de control (resto de entidades). Dicha técnica permite obtener resultados del grupo de tratamiento antes de la reforma a la ley, así como después de su entrada en vigor, lo cual agrega robustez al ejercicio porque compara el grupo de tratamiento en dos momentos en el tiempo. Esto último permite distinguir a este trabajo del resto que han sido elaborados para tal objetivo, debido a que en la mayoría son evaluados los efectos de la reforma a través de análisis con datos de corte transversal.

En algunos trabajos han sido evaluados aspectos relacionados con la homologación del IVA en la frontera norte, Fuentes, Ruiz y Brugués (2014); Ruiz y Brugués (2014); Robles, Mendoza, Palomino y Ramírez (2015); Fuentes, Ruiz, González y Brugués (2016), sobre todo desde una perspectiva del efecto sobre los precios y la dinámica económica de la frontera, algunos han tocado el aspecto del consumo de los hogares y las pérdidas de bienestar, no obstante, el método más utilizado toma en cuenta datos de corte transversal, con la limitante de no poder comparar contra un periodo anterior el efecto de la homologación del IVA.

La organización del artículo es la siguiente: La primera sección contiene la revisión de la literatura, posteriormente es presentada la metodología que permite llevar a cabo la evaluación aquí propuesta, posteriormente se encuentra el apartado de los resultados. Finalmente son presentadas las reflexiones finales en donde también son planteadas algunas recomendaciones de política pública relacionadas con el IVA y el consumo en Baja California, Baja California Sur y Quintana Roo.

\section{Revisión de la literatura.}

La primera ley del IVA fue decretada el 23 de noviembre de 1978, ahí se estipulaba que la tasa del IVA para la zona fronteriza de los estados del norte, así como para las zonas libres de Baja California y Baja California Sur, sería del 6\%. La finalidad de diferenciar la tasa de este impuesto en ese momento era homologar dicho impuesto con el que se cobraba del otro lado de la frontera para evitar que los residentes de México cruzaran la frontera para comprar en los Estados Unidos (EE. 
UU.) (Davis 2011). Lo anterior estuvo vigente hasta 1992 cuando el gobierno decidió incrementar la tasa al 10\% mientras en el resto del país quedó fijada en 15\%, además cambió la delimitación de la región fronteriza, considerando en ese entonces el territorio que comprende la franja de $20 \mathrm{~km}$ paralela a las líneas divisorias internacionales del norte y sur del país, todo el territorio de los estados de Baja California, Baja California Sur y Quintana Roo (BC, BCS y QROO), los municipios de Caborca y Cananea, Sonora, así como la región parcial del estado de Sonora que comprende otros municipios no fronterizos (Ley del Impuesto al Valor Agregado 2009). Durante los próximos 17 años estuvo vigente esta ley, hasta el año 2009, cuando se incrementaron ambas tasas, quedando el IVA de la región fronteriza en 11\%, mientras en el resto del país en 16\%. El objetivo de tener esta tasa diferenciada era mantener la competitividad a través de los precios de la región fronteriza respecto a los estados de los EE. UU. cuyo impuesto al valor agregado es alrededor de $8 \%$, además de reducir los costos de distribución que implica el aislamiento de la región de los principales centros de abasto del país (Fuentes, Ruiz y González, y otros 2016).

En 2013, el gobierno federal llevó a cabo una reforma fiscal que entró en vigor el $1^{\circ}$ de enero de 2014, entre otros aspectos, consideró la eliminación del trato diferenciado en la tasa del IVA en la región fronteriza, homologando la tasa con el resto del país, pasando del $11 \%$ al 16\% de un año a otro.

Uno de los argumentos que el gobierno federal utilizó para justificar la eliminación del trato diferenciado del IVA en la región fronteriza, fue que el ingreso por habitante en dicha región es superior en 27\% con respecto al resto del país (Gobierno de la República 2013), no obstante, el indicador utilizado para sustentar este argumento es el Producto Interno Bruto per cápita (PIBpc), el cual está influenciado por el efecto de la actividad turística en los estados de Baja California Sur y Quintana Roo, lo cual eleva de manera considerada el indicador y su utilización en este contexto es cuestionable.

Otro argumento utilizado para justificar la homologación del IVA es que el beneficio de la menor tasa no llega a los consumidores de esta región, sino que beneficia a los productores y comerciantes. El Gobierno de la República (2013) utiliza como ejemplo los precios de los muebles y de los electrodomésticos, los cuales afirma, sin citar fuente, que son 4\% más caros en la región fronteriza que en el resto del país. En este mismo sentido, en el citado documento se asegura que los artículos de higiene y cuidado personal son 2\% más caros en la región fronteriza que en el resto del país, y en promedio la diferencia de precios es $2 \%$ más caros en donde se le da un trato diferenciado a la tasa del IVA.

Si los productos en la región fronteriza son en promedio más caros, no puede afirmarse que es consecuencia de que los productores y comerciantes absorban los beneficios de la menor tasa de IVA, ya que en el documento del Gobierno de la República (2013) jamás se demuestra esto, aunque 
tampoco se pone a prueba la hipótesis de que los costos de transporte encarecen los productos que son distribuidos en la mencionada región, ya que una de las causas de la tasa diferenciada de IVA para la región fronteriza era el aislamiento de dicha región con respecto al resto del país (Gobierno de la República 2013). Uno de los principales centros productores del país está localizado en la Zona Metropolitana del Valle de México, de donde son abastecidos algunos bienes del mercado de la región fronteriza.

La teoría asegura que un impuesto generalizado como el IVA que es aplicado a todos los bienes que son intercambiados en la economía, generará un efecto creciente sobre los precios, a su vez este efecto ocasionará efectos sobre el consumo por dos vías; 1) Absorbe el poder adquisitivo que puede destinarse tanto para consumo o para ahorro 2) Penaliza el gasto en consumo, mientras no genera un desaliento inmediato para ahorrar (Shultz 1943). Es esperado que estos efectos impacten con mayor fuerza a los deciles de más bajos ingresos o ingresos medios, debido a que la mayor parte de sus ingresos está destinada al consumo. Es necesario considerar que en México los alimentos están exentos del pago de IVA, sin embargo, el impacto esperado en el consumo es indirecto, debido a que los bienes y servicios utilizados para la distribución de alimentos cuenta con gravamen de IVA, por esta razón, mientras los salarios no se recuperen, el efecto de elevar este impuesto perdurará en el consumo y en las ventas (Fuentes, Ruiz y González, y otros 2016).

Más allá de los beneficios que la política de diferenciación acarreaba para las empresas y para la región en general, los consumidores tenían acceso a productos con precios competitivos lo cual se ve reflejado en la mayor demanda de bienes y servicios. En consecuencia, el encarecimiento indirecto de los bienes a través de un aumento en la tasa del IVA puede tener consecuencias negativas en el consumo de los hogares de la región fronteriza, que puede repercutir en el bienestar de estos, además está presente el denominado "problema de la ciudad fronteriza", el cual surge cuando un incremento o imposición de un impuesto en una jurisdicción o región crea un incentivo en los consumidores para sustituir el lugar de altos impuestos por el lugar de bajos impuestos para llevar a cabo sus compras (Fisher 1980). Este fenómeno se agudiza cuando el impuesto en cuestión se establece sobre las ventas, sobre productos seleccionados, sobre la propiedad o sobre el ingreso (Fox 1986). De manera general, cualquier impuesto modifica las decisiones de consumo, producción e inversión de los agentes económicos, porque altera los precios relativos, tanto de los insumos como de los bienes y servicios finales. Las exenciones, como la tasa preferencial en la región fronteriza juegan a favor del contribuyente ya que pueden reflejarse en menores precios al público (Anselmo 2007). En específico para la frontera norte de México, es esperada una sustitución de productos locales de alta elasticidad por productos importados (Fuentes, Ruiz y Brugués 2014) que pueden ser productos primarios o manufacturados. Este fenómeno es corroborado por Corrales y González (2017), quienes hallan una pérdida de competitividad de algunos sectores del comercio al por menor, producto de la fuga de consumidores que deciden sustituir el consumo local por las importaciones. 


\section{○ 3. Metodología.}

Con $n$ bienes, el objetivo del individuo consiste en maximizar la utilidad que obtiene de estos bienes (Nicholson 2008),

$$
\text { utilidad } \equiv U=U\left(x_{1}, x_{2}, \ldots x_{n}\right),
$$

Sujeta a la restricción presupuestaria

$$
I=p_{1} x_{1}+p_{2} x_{2}+\cdots+p_{n} x_{n}
$$

En donde cada $p$ es el precio que se paga por el bien x. La suma de los gastos de los bienes es igual al gasto total que por simplicidad se asume que es igual al ingreso I. Es decir, supone que todo el ingreso se gasta, de tal manera que,

$$
I-p_{1} x_{1}-p_{2} x_{2}-\cdots-p_{n} x_{n}=0
$$

Por lo tanto, la función de maximización puede expresarse como

$$
\prod^{n}=U\left(x_{i}\right)+\gamma\left(I-\sum^{n} p_{i} x_{i}\right)
$$

De lo anterior se deduce que la utilidad marginal del bien i está relacionada de manera negativa con el precio de esta, es decir,

$$
\frac{\partial \pi}{x_{i}}=\frac{\partial U\left(x_{i}\right)}{x_{i}}-\gamma p_{i}
$$

A su vez, el precio $p$ del bien i está compuesto por $(p+t)$, en donde t es el impuesto que se paga por el bien $x$. Lo cual implica que un aumento en el precio o en el impuesto disminuye la utilidad de ese bien siempre y cuando el ingreso disponible no aumente.

De tal forma que, al considerar que los gustos y preferencias que maximizan la utilidad marginal ante el consumo de un bien no cambian de un periodo a otro, la diferencia en el consumo debe explicarse por la descomposición de los gastos totales en cada uno de los bienes, por lo que, manteniendo todos los factores constantes, un cambio en el consumo de un bien está relacionado de manera directa con un cambio en el precio de este.

Con base en lo anterior, el consumo de bienes está relacionado de manera inversa con el precio de estos y de manera positiva con el ingreso, de igual manera existen otros factores que intervienen en las decisiones de consumo tales como 1) gustos 2) expectativas 3) número de compradores (Mankiw 2012). Al tratarse de hogares, los gustos y las expectativas pueden aproximarse con la edad 
del jefe del hogar y con sus años de escolaridad. Por otra parte, el número de compradores, como determinante del consumo, la cantidad de integrantes del hogar, lo cual evita un posible efecto de escalamiento, debido a que un hogar numeroso puede consumir más que un hogar compuesto por un individuo (McCracken y Brandt 1987) (Wang, Sindelar y Busch 2006).

Esto establece que el gasto en un bien determinado dependa de cuatro factores,

$$
G_{i}=f\left(I_{i}, E_{i}, D_{i}, T_{i}\right)
$$

En donde,

$G_{i}=$ Gasto del hogar $i$ en el bien $x$

$I_{i}=$ Ingreso del hogar $i$

$E_{i}=$ Edad del jefe del hogar $i$

$D_{i}=$ Educación del jefe del hogar $i$

$T_{i}=$ Número de ingrantes del hogar $i$

Para evaluar el impacto de la reforma a la ley del IVA en el consumo de los hogares fronterizos es considerada una técnica cuasi experimental, debido a que el cambio esperado en el comportamiento del consumo de los hogares es una consecuencia de un cambio en un factor incontrolable por estos, es decir, un evento exógeno, como lo es un cambio en la política fiscal. Como tal, un cuasiexperimento está caracterizado por tener un grupo de control, que no está expuesto al cambio en la decisión de gobierno, así como el grupo de tratamiento, que es el que se ve afectado por dicho cambio exógeno.

Sea $C$ el grupo de control y sea $T$ el grupo de tratamiento, sea $d T$ una variable binaria que identifica el grupo de tratamiento asignando el valor de 1 si pertenece al conjunto de observaciones sujetas al cambio en la política pública y cero para el resto. Por su parte, sea $d 2$ una variable binaria que identifica el segundo periodo o el periodo que contiene las observaciones una vez entrada en vigor la nueva política, con lo anterior es definida la siguiente ecuación

$$
y=\beta_{0}+\delta_{0} d 2+\beta_{1} T+\delta_{1} d 2 \cdot d T+\boldsymbol{X} \boldsymbol{\beta}+\varepsilon
$$

En donde y es la variable de interés, esa que es afectada por el cambio en la política pública, por su parte, $X$ es un conjunto de variables explicativas del comportamiento de la variable dependiente, $\varepsilon$ es un término de error estocástico. El parámetro de interés es $\delta 1$, denominado el estimador de diferencia en diferencias (DD), el cual mide el efecto de la política (Wooldridge 2016). Este estimador, 
puede expresarse como:

$$
\hat{\delta}_{1}=\left(\bar{y}_{2, T}-\bar{y}_{2, C}\right)-\left(\bar{y}_{1, T}-\bar{y}_{1, C}\right)
$$

En donde el operador barra indica el promedio, el primer subíndice el periodo y el segundo subíndice el grupo, lo cual significa que el estimador de diferencia en diferencias evalúa el impacto sobre los valores medios de la variable de interés.

La metodología aquí propuesta ha sido catalogada como una de las herramientas cuasi experimentales más efectivas para evaluar efectividad en la implementación de política pública para los EE. UU. (Handley, y otros 2018), así como uno de los diseños metodológicos más eficientes para evaluar las mejores prácticas en la salud pública (Wing, Simon y Bello-Gómez 2018).

Esta técnica ha sido utilizada para medir el efecto de medidas de seguridad en las tasas de criminalidad en la frontera de algunos países de Europa del este en Sander y Wassmann (2017), también ha sido implementada por Yonas y Broussard (2018) para medir el impacto en la productividad de unos agricultores en Etiopía que se inscriben en un programa de mejora tecnológica para la producción.

Con base en lo anterior, la especificación econométrica queda como sigue:

$$
G_{i t .}=\beta_{0}+\delta_{0} d 14_{i t}+\beta_{1} F_{i t .}+\delta_{1} d 14_{i t .} \cdot F_{i t}+\beta_{2} I_{i t .}+\beta_{3} E_{i t}+\beta_{4} D_{i t .}+\beta_{4} T_{i t}+\varepsilon_{i t}
$$

En donde d14 es una variable binaria para identificar las observaciones del año 2014, $F_{i}$ es una variable binaria para identificar a las entidades federativas de Baja California, Baja California Sur y Quintana $\operatorname{Roo}^{1}, d 14_{i} \cdot F_{i}$ son las observaciones de las tres entidades federativas citadas en el año 2014. El resto de las variables fueron explicadas párrafos atrás.

Para la estimación se dispone de datos de la Encuesta Nacional de Ingresos y Gastos de los Hogares (ENIGH) de los años 2012 y 2014, la cual incluye la información necesaria para llevar a cabo el experimento. Los años en que se tiene la información disponible son apropiados para el objetivo del presente documento, debido a que la homologación del IVA en la región fronteriza entró en vigor a partir del 1 de enero de 2014.

La ENIGH proporciona información sobre el gasto en diferentes bienes, sobre todo aquellos que conforman la canasta básica, entre otros, por lo que es posible extender el mismo análisis desagre-

\footnotetext{
${ }^{1} \mathrm{Si}$ bien se conoce que la región fronteriza está conformada por más municipios de otras entidades, los tres estados considerados son los únicos en donde todo el territorio forma parte de la región fronteriza. En el resto de los casos, existen municipios que pertenecen a la entidad, pero no forman parte de la región fronteriza y la ENIGH no es representativa al nivel municipal.
} 
gándolo para cada uno de los conceptos de gasto en diversos bienes para evaluar en cuáles repercutió de manera significativa la reforma al IVA. Para llevar a cabo este experimento el gasto en alimentos es excluido, debido a que estos están exentos de cualquier impuesto, por lo tanto, la cantidad de bienes que son evaluados en este trabajo son 25 .

Las ENIGH contienen información del gasto y del ingreso corrientes en el año que son levantadas las encuestas, por esta razón es necesario deflactar todas las variables monetarias para que la comparación temporal tenga sentido, para esto es utilizado el Índice Nacional de Precios al Consumidor (INPC) base 2008 que proporciona el INEGI. De igual manera, para el gasto de cada bien fue utilizado el INPC por concepto del gasto, con el objetivo de calcular con mayor precisión el gasto real.

\section{$\rightarrow$ 4. Resultados.}

A continuación, en el cuadro 1, se muestran estadísticos descriptivos del gasto trimestral de los 25 bienes que son evaluados para las tres entidades federativas bajo estudio, considerando el año 2012 y el 2014. En este cuadro se muestran el promedio, la desviación estándar y el porcentaje de hogares con cero consumos en cada uno de los bienes. La mayoría de los bienes reportan un consumo menor en 2014 con respecto a 2012, solo el consumo de agua, hospitalización y educación tienen un consumo promedio mayor en 2014.

A través del porcentaje de hogares con cero consumos en los bienes, puede tenerse una idea sobre la distribución del gasto. Por ejemplo, el gasto en bienes del cuidado del hogar, así como del cuidado personal es frecuentado en 99\% de los hogares en México, ya que solo 1\% de estos reporta un gasto igual a cero. En contraparte, solo 2\% de los hogares en México gastan en hospitalización, ya que $98 \%$ reporta un gasto igual a cero en este rubro.

Esta asimetría en los datos tiene implicaciones para el método de estimación, debido a que en unos bienes se tienen más datos diferentes de cero que en otros. Cuando los datos tienen esta característica el método más apropiado de estimación es a través de un modelo Tobit (Wooldridge 2016). 


\begin{tabular}{|c|c|c|c|c|c|c|}
\hline \multicolumn{7}{|c|}{$\begin{array}{c}\text { Cuadro } 1 \\
\text { Estadísticos descriptivos del gasto por bien en Baja California, } \\
\text { Baja California Sur y Quintana Roo } \\
2012-2014\end{array}$} \\
\hline \multirow{2}{*}{ Variable } & \multicolumn{2}{|c|}{ Promedio } & \multicolumn{2}{|c|}{ Desv. Estándar } & \multicolumn{2}{|c|}{ Obs. $=0$} \\
\hline & 2012 & 2014 & 2012 & 2014 & 2012 & 2014 \\
\hline Bebidas & $\$ 876$ & $\$ 805$ & $\$ 1,119$ & $\$ 810$ & $11 \%$ & $9 \%$ \\
\hline Alimentos fuera hogar & $\$ 2,169$ & $\$ 1,863$ & $\$ 3,837$ & $\$ 3,967$ & $46 \%$ & $54 \%$ \\
\hline Tabaco & $\$ 56$ & $\$ 43$ & $\$ 298$ & $\$ 275$ & $94 \%$ & $95 \%$ \\
\hline Vestido & $\$ 1,434$ & $\$ 1,147$ & $\$ 4,007$ & $\$ 1,778$ & $27 \%$ & $22 \%$ \\
\hline Agua & $\$ 400$ & $\$ 410$ & $\$ 474$ & $\$ 662$ & $17 \%$ & $21 \%$ \\
\hline Energía eléctrica & $\$ 1,460$ & $\$ 1,349$ & $\$ 1,841$ & $\$ 1,541$ & $9 \%$ & $9 \%$ \\
\hline Cuidado hogar & $\$ 1,326$ & $\$ 1,078$ & $\$ 2,448$ & $\$ 1,683$ & $1 \%$ & $1 \%$ \\
\hline Utensilios & $\$ 97$ & $\$ 78$ & $\$ 338$ & $\$ 370$ & $76 \%$ & $79 \%$ \\
\hline Enseres & $\$ 283$ & $\$ 194$ & $\$ 1,264$ & $\$ 625$ & $73 \%$ & $71 \%$ \\
\hline Salud & $\$ 601$ & $\$ 423$ & $\$ 2,800$ & $\$ 2,145$ & $58 \%$ & $57 \%$ \\
\hline Atención médica & $\$ 481$ & $\$ 219$ & $\$ 2,694$ & $\$ 1,012$ & $72 \%$ & $73 \%$ \\
\hline Hospitalización & $\$ 23$ & $\$ 119$ & $\$ 264$ & $\$ 1,713$ & $98 \%$ & $98 \%$ \\
\hline Transporte público & $\$ 860$ & $\$ 850$ & $\$ 1,702$ & $\$ 1,543$ & $61 \%$ & $56 \%$ \\
\hline Transporte foráneo & $\$ 223$ & $\$ 213$ & $\$ 814$ & $\$ 1,214$ & $84 \%$ & $86 \%$ \\
\hline Adquisición vehículo & $\$ 897$ & $\$ 559$ & $\$ 6,957$ & $\$ 4,537$ & $94 \%$ & $95 \%$ \\
\hline Mantenimiento vehículo & $\$ 2,550$ & $\$ 1,965$ & $\$ 3,567$ & $\$ 2,930$ & $37 \%$ & $45 \%$ \\
\hline Refacciones & $\$ 197$ & $\$ 151$ & $\$ 683$ & $\$ 562$ & $77 \%$ & $79 \%$ \\
\hline Combustible & $\$ 2,353$ & $\$ 1,814$ & $\$ 3,393$ & $\$ 2,723$ & $38 \%$ & $46 \%$ \\
\hline Comunicación & $\$ 1,516$ & $\$ 1,091$ & $\$ 1,739$ & $\$ 1,477$ & $17 \%$ & $22 \%$ \\
\hline Educación & $\$ 1,825$ & $\$ 1,846$ & $\$ 5,539$ & $\$ 5,199$ & $63 \%$ & $62 \%$ \\
\hline Esparcimiento & $\$ 982$ & $\$ 723$ & $\$ 2,222$ & $\$ 1,515$ & $40 \%$ & $45 \%$ \\
\hline Paquetes turísticos & $\$ 126$ & $\$ 97$ & $\$ 811$ & $\$ 870$ & $95 \%$ & $97 \%$ \\
\hline Cuidado personal & $\$ 1,585$ & $\$ 1,406$ & $\$ 1,605$ & $\$ 1,464$ & $1 \%$ & $1 \%$ \\
\hline Accesorios personales & $\$ 73$ & $\$ 41$ & $\$ 355$ & $\$ 179$ & $80 \%$ & $83 \%$ \\
\hline Otros gastos & $\$ 315$ & $\$ 198$ & $\$ 1,469$ & $\$ 1,213$ & $87 \%$ & $90 \%$ \\
\hline
\end{tabular}

Fuente: Elaboración propia con datos de la ENIGH 2012 y 2014.

En el cuadro 2, por su parte, se muestran estadísticos descriptivos de las variables independientes, además del gasto total en las entidades de interés para los dos años que están comparándose.

El gasto total promedio disminuye 16\% en 2014 con respecto a 2012. El gasto mínimo no reporta un cambio significativo, no obstante, la diferencia en el gasto máximo de un año con respecto al otro es considerable, ya que en términos porcentuales disminuye 92\%, lo que explica la disminución del gasto total promedio.

Por otro lado, el ingreso disminuye 11\% en 2014 con respecto a 2012, sin embargo, en este caso el ingreso mínimo es mayor en 2014. De la misma manera, el ingreso máximo es 1.4\% superior en 2014.

El resto de las variables, como la edad y educación del jefe del hogar no exhiben diferencias 
sustanciales entre los dos años, al igual que el total de integrantes del hogar, cuyos valores medios y mínimos se mantienen constantes.

Cuadro 2

Estadísticos descriptivos del gasto total, ingreso, edad del jefe del hogar, educación del jefe del hogar y total de integrantes del hogar

\begin{tabular}{lcccccccc}
\hline \multirow{2}{*}{ Variable } & \multicolumn{2}{c}{ Promedio } & \multicolumn{2}{c}{ Desv. Estándar } & \multicolumn{2}{c}{ Min } & \multicolumn{2}{c}{ Max } \\
& 2012 & 2014 & 2012 & 2014 & 2012 & 2014 & 2012 & 2014 \\
\hline Gasto Total & $\$ 28,019$ & $\$ 24,006$ & $\$ 26,510$ & $\$ 20,516$ & $\$ 0$ & $\$ 79$ & $\$ 405,908$ & $\$ 210,705$ \\
Ingreso & $\$ 43,786$ & $\$ 39,260$ & $\$ 39,330$ & $\$ 33,087$ & $\$ 1,140$ & $\$ 2,637$ & $\$ 368,539$ & $\$ 374,069$ \\
Edad del jefe & 47 & 44 & 16 & 14 & 18 & 16 & 97 & 91 \\
Educación del jefe & 6 & 6 & 3 & 2 & 1 & 1 & 11 & 11 \\
Integrantes del hogar & 4 & 4 & 2 & 2 & 1 & 1 & 16 & 14 \\
\hline
\end{tabular}

Fuente: Elaboración propia con datos de la ENIGH 2012 y 2014.

En el cuadro 3, se muestra el consumo por año y por grupo, es decir, si pertenece a las tres entidades bajo estudio o no. En el cuadro puede observarse que las entidades no consideradas en la región fronteriza tuvieron un incremento en el consumo promedio de 20,934 a 21,734, lo que representa un incremento de 4\% de 2012 a 2014. Por otro lado, las entidades de BC BCS y QROO pasaron de un consumo de 28,019 a uno de 24,006 en promedio, lo cual representa una disminución de 14\%.

\begin{tabular}{|c|c|c|c|c|}
\hline & $\mathrm{BC}, \mathrm{BCS}$ & QROO & & \\
\hline Año & No & Sí & & Total \\
\hline & \$ 20,934.19 & $\$ 28,019.26$ & $\$$ & $21,570.13$ \\
\hline 2012 & $\$ 23,288.85$ & $\$ 26,510.10$ & $\$$ & $23,681.09$ \\
\hline & 8,194 & 808 & & 9,002 \\
\hline & $\$ 21,734.44$ & $\$ 24,006.21$ & $\$$ & $21,921.86$ \\
\hline 2014 & $\$ 23,227.37$ & $\$ 20,516.21$ & $\$$ & $23,023.80$ \\
\hline & 17,872 & 1,607 & & 19,479 \\
\hline & $\$ 21,482.88$ & $\$ 25,348.88$ & $\$$ & $21,810.69$ \\
\hline Total & $\$ 23,249.24$ & $\$ 22,771.84$ & $\$$ & $23,233.72$ \\
\hline & 26,066 & 2,415 & & 28,481 \\
\hline
\end{tabular}

Fuente: Elaboración propia con datos de la ENIGH 2012 y 2014. 
En el año 2012, BC, BCS y QROO tenían un consumo promedio 34\% mayor que el resto de las entidades, mientras en 2014 este mismo rubro era 10\% mayor.

En el cuadro 4 se muestran los mismos estadísticos descriptivos que el cuadro anterior, pero ahora de la variable que contiene el ingreso de los hogares. El cuadro 4 revela que, en términos de ingreso, las entidades de BC, BCS y QROO están por encima del resto del país, sin embargo, debe notarse que, de 2012 a 2014 tuvo una disminución porcentual de 10\%, mientras en el resto del país el ingreso de los hogares incrementó 5\%. En 2012, el ingreso de los hogares de la región fronteriza era 42\% más que en el resto de los hogares, mientras en 2014 el mismo indicador es 22\% mayor.

\begin{tabular}{ccccc}
$\begin{array}{c}\text { Media, desviación estándar y frecuencia del ingreso } \\
\text { por año y grupo }\end{array}$ \\
\hline \multicolumn{5}{c}{ BC, BCS y QROO } \\
\hline Año & No & Sí & & Total \\
& & & & \\
\multirow{4}{*}{$\mathbf{2 0 1 2}$} & $\$ 30,823.29$ & $\$ 43,785.55$ & $\$$ & $31,986.76$ \\
& $\$ 37,080.14$ & $\$ 39,329.73$ & $\$$ & $37,468.98$ \\
& 8,194 & 808 & & 9,002 \\
\multirow{2014}{*}{ Total } & $\$ 46,770.25$ & $\$ 33,087.18$ & $\$$ & $45,836.07$ \\
& 17,872 & 1,607 & & 19,479 \\
& $\$ 43,959.37$ & $\$ 35,355.28$ & $\$$ & $43,367.51$ \\
& 26,066 & 2,415 & & 28,481 \\
\hline
\end{tabular}

Fuente: Elaboración propia con datos de la ENIGH 2012 y 2014.

Con base en los datos, debe considerarse que tanto el consumo y el ingreso contienen valores cero en algunas observaciones, lo cual condiciona la posibilidad de calcular el logaritmo natural de dicha variable, de lo contrario implicaría que esas observaciones no sean consideradas. Esto implica que la estimación deba hacerse en niveles, no obstante, también debe tenerse en cuenta que el método de mínimos cuadrados ordinarios pierde eficiencia cuando las variables no están distribuidas de forma normal, por esta razón es utilizada la regresión de Tobit para llevar a cabo el análisis.

Son estimadas 25 ecuaciones, cada una corresponde a un componente del consumo de los hogares, los coeficientes de cada estimación son mostradas en los anexos, debido a que, por una parte, éstos no deben interpretarse de manera directa, ya que la estimación se hace sobre una variable latente $\mathrm{y}^{\wedge *}$, y lo que interesa es el efecto marginal promedio del coeficiente de DD. En la gráfica 1 es mostrado el efecto marginal promedio del estimador de DD para cada uno de los 25 bienes evaluados. 


\section{Estimador de diferencia en diferencias por concepto de consumo}

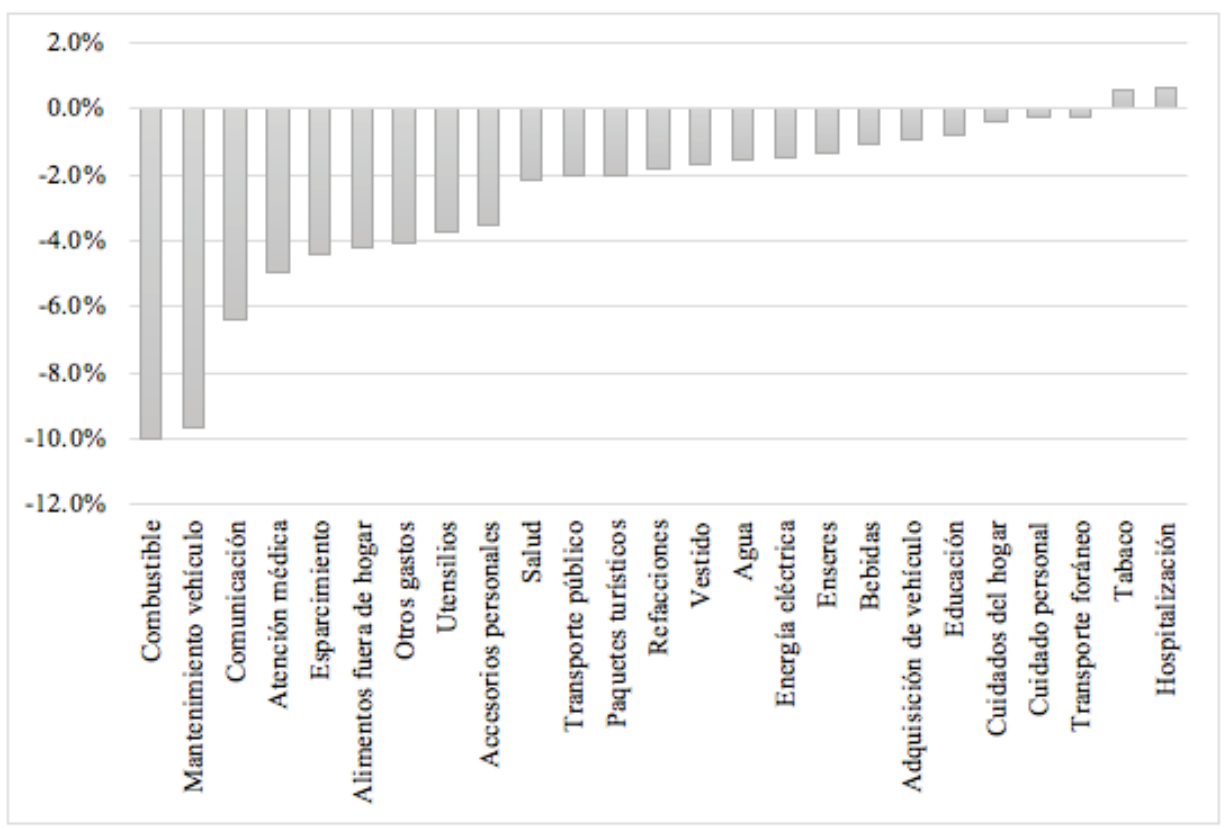

Fuente: Estimaciones propias.

La gráfica anterior revela que la reforma a la ley del IVA de 2013 tuvo mayor impacto en el consumo de combustible, con una disminución promedio de 10\%. Las personas con acceso al mercado estadounidense, es decir, aquellas que tienen visa para ingresar a territorio de los EE. UU., tienen la posibilidad de decidir entre consumir en México o en el país vecino. Si bien está prohibida la importación de combustible, salvo aquel que contenga el tanque de gasolina del vehículo, esto facilita que las personas con posibilidad de cruzar la frontera se abastezcan de combustible en los EE. UU. y sustituyan el consumo local de este bien. Con excepción de tabaco y hospitalización, el impacto en el resto de los componentes del consumo de los hogares de la región fronteriza fue negativo. 


\section{D.Reflexiones finales.}

En el presente ejercicio es evaluada la reforma a la ley del IVA de 2013 que entró en vigor el 01 de enero de 2014. Tal como la teoría lo estipula, un aumento o la imposición de algún impuesto generalizado a los bienes tendrá repercusiones negativas en el consumo de estos, en el presente trabajo no ha podido rechazarse dicha afirmación, debido a que el consumo de los hogares de los estados de Baja California, Baja California Sur y Quintana Roo se contrajo en promedio $11.4 \%$ una vez entrada en vigor la mencionada reforma. De manera particular, 23 de 25 diferentes conceptos de consumo sufrieron un impacto negativo, lo cual repercute en el bienestar de los hogares. Los resultados aquí mostrados permiten contradecir con amplia confianza los argumentos planteados para justificar elevar la tasa del IVA en la región fronteriza por parte del Gobierno de la República, el presente estudio demuestra que la reforma al IVA afectó a los consumidores finales, en consecuencia, también a los productores y comerciantes que llevan a cabo sus actividades en esta región.

El "problema de la ciudad fronteriza" emerge en el caso mexicano, los hogares fronterizos, de manera particular los del norte del país, tienen acceso a comprar bienes en el mercado estadounidense, por lo que la sustitución de compras locales por importaciones es una afirmación razonable, esto implica una disminución de consumo local, lo que a su vez tiene repercusiones en el comercio al por menor, lo cual deteriora la dinámica económica local.

Este tipo de efectos son indeseables para cualquier hogar y para cualquier gobierno, no obstante, por tratarse de hogares fronterizos, tienen la posibilidad de importar los bienes que de manera local se encarecen, sin embargo, esto no ha podido corroborarse con el presente trabajo. En su defecto, los oferentes de bienes y servicios locales se ven afectados si el mercado local decide comprar en el exterior bienes que en el pasado compraba en el mercado nacional.

Cabe señalar que los bienes que sufrieron un mayor impacto negativo están ligados de manera directa e indirecta a los procesos de distribución de bienes y servicios, tales como el combustible, mantenimiento de vehículo y comunicación, unas de las justificaciones para mantener la tasa del IVA diferenciada en la región fronteriza. No es un caso de infraestructura, tal como fue estipulado en Gobierno de la República (2013), sino el precio que se paga por esta, el componente territorial debe de ser tomado en cuenta, ya que la propia irregularidad del territorio demarca las distancias que tienen que recorrerse para llevar productos del centro y sur del país hacia el norte o hacia el sur-este del mismo.

Ante la nueva administración de los EE. UU., los hogares fronterizos también han experimentado el encarecimiento indirecto de las importaciones por la depreciación de la moneda nacional ante el dólar estadounidense. Cabe señalar que esta situación jugó a favor de una menor sustitución de consumo local, debido a que, por el tipo de cambio elevado, los bienes en el extran- 
jero se encarecieron, dejando en un impase a los hogares fronterizos.

Una implicación de política pública derivada del presente documento es el regreso de la tasa preferencial de IVA para la región fronteriza, de tal manera que pueda restaurarse el consumo de los hogares, incluso una tasa más baja que la anterior impulsaría los incentivos para consumir e invertir en la región, debido a que los mismos productos podrían abaratarse para los extranjeros, quienes podrían cruzar a México para consumir. En términos absolutos es un mercado más grande que podría aprovecharse, además que cualquier ciudadano estadounidense puede ingresar a territorio mexicano debido a que no les es requerida una visa para esto. El caso de Quintana Roo no es la excepción, población de Belice puede ingresar a territorio nacional para consumir bienes y servicios si estos son abaratados de manera indirecta a través de una tasa impositiva preferencial. 


\section{Bibliografía y fuentes documentales}

Anselmo, Fernando. «Propuesta para homologar las tasas del Impuesto al Valor Agregado con base en los principios universales de los impuestos.» Tesis de Maestría, Facultad de Contaduría y Administración, Querétaro, Querétaro, 2007.

Corrales, Salvador, y Salvador González. «Comercio al menudeo y reforma fiscal en la frontera México-Estados Unidos.» Trayectorias 19, nº 44 (2017): 64-85.

Davis, Lucas W. «The effects of prefferential VAT rates near international borders: Evidence from Mexico.» National Tax Journal 64, $\mathrm{n}^{\circ} 1$ (2011): 85-104.

Diario Oficial de la Federación. «Ley del Impuesto al Valor Agregado.» 1 de Enero de 2009: 1-89.

Fisher, Ronald C. «Local sales taxes: Tax rates differentials, sale loss, and revenue estimation.» Public Finance Quarterly 8, nº 2 (1980): 171-188.

Fox, William F. «Tax structure and the location of economic activity along state borders.» National Tax Journal 39, no 4 (1986): 387-401.

Fuentes, Noé Arón, Wilfrido Ruiz, Gabriel González, y Alejandro Brugués. «Exploración de los impactos de la homologación del IVA en la región y franja fronteriza, con particular referencia al caso de Baja California.» El Cotidiano, no 195 (2016): 63-74.

Fuentes, Noé Arón, Wilfrido Ruiz, y Alejandro Brugués. «Explorando los Efectos Económicos Previstos de la Homologación del IVA en la Región y Franja Fronteriza: El caso de Baja California.» Economía Actual 7, no 4 (2014): 31-36.

Fuentes, Noé Arón, Wilfrido Ruiz, y Alejandro Brugués. «Explorando los Efectos Económicos Previstos de la Homologación del IVA en la Región y Franja Fronteriza; el caso de Baja California.» Economía Actual 7, no 4 (2014): 31-36.

Gobierno de la República. «Criterios generales de política económica 2014.» México, 2013.

Handley, Margaret A., Courtney R. Lyles, Charles McCulloch, y Adithya Cattamanchi. «Selecting and Improving Quasi-Experimental Designs in Effectiveness and Implementation Research.» Annual Reviews, 2018: 26.1-26.21.

Mankiw, N. Gregory. Principios de Economía. Sexta. Editado por Javier Reyes y Timoteo Eliosa. D.F.: Cengage Learning, 2012.

McCracken, Vicki A, y Jon A. Brandt. «Household Consumption of Food-Away-From-Home: Total expenditure and by Type of Food Facility.» The American Journal of Agricultural Economics, 1987: 275-284.

Nicholson, Walter. Teoría Microeconómica. Principios básicos y ampliaciones. 9a. México: Cengage Learning, 2008.

Robles, José Ezequiel, Fidel Antonio Mendoza, Rossana Palomino, y Sergio Ramiro Ramírez. «Homologación del IVA 2014 en la frontera norte de México: Inflación y reordenamiento económico.» Global Conference on Business and Finance Proceedings. Las Vegas, 2015. 822-828.

Ruiz, Wilfrido, y Alejandro Brugués. «Primer Boletín del Observatorio de Coyuntura de la Economía Fronteriza.» El Ecolegio de la Frontera Norte, Tijuana, 2014.

Sandner, Malte, y Pia Wassmann. «The Effect of Changes in Border Regimes on Border Regions Crime Rates: Evidence from the Schengen Treaty.» Center for Research and Analysis of Migration, 2017: $1-30$.

Shultz, William J. «Economic Effects of a Federal General Sales Tax.» The Tax Magazine 21, nº 8 (1943): 419-439.

Wang, Hong, Jody L. Sindelar, y Susan H. Busch. «The impact of tobacco expenditure on household consumption patterns in rural China.» Social Science \& Medicine, no 62 (2006): 1414-1426. 
Wing, Coady, Kosali Simon, y Ricardo A. Bello-Gómez. «Designing Difference in Differences Studies: Best Practices for Public Health Policy Research.» Annual Reviews, 2018: 29.1-29.17.

Wooldridge, Jeffrey M. Introductory Econometrics: A modern approach. 6th. Boston, MA: Cengage Learning, 2016.

Yonas, Alem, y Nzinga H. Broussard. «The impact of safety nets on technology adoption: a difference-in-differences analysis.» Agricultural Economics, nº 49 (2018): 13-24. 


\section{Anexo 1}

\section{Regresiones Tobit por bien de consumo}

\begin{tabular}{|c|c|c|c|c|c|c|c|}
\hline VARS & $\begin{array}{c}\text { (1) } \\
\text { Bebidas }\end{array}$ & $\begin{array}{c}\text { (2) } \\
\begin{array}{c}\text { Alimentos fuera del } \\
\text { hogar }\end{array}\end{array}$ & $\begin{array}{c}\text { (3) } \\
\text { Tabaco }\end{array}$ & $\begin{array}{c}\text { (4) } \\
\text { Vestido }\end{array}$ & $\begin{array}{l}\text { (5) } \\
\text { Agua }\end{array}$ & $\begin{array}{c}\text { (6) } \\
\text { Energia }\end{array}$ & $\begin{array}{c}\text { (7) } \\
\text { Cuidado hogar }\end{array}$ \\
\hline F & $\begin{array}{c}248.145 * * * \\
(33.913)\end{array}$ & $\begin{array}{c}61.705 \\
(180.238)\end{array}$ & $\begin{array}{c}-243.480 \\
(151.624)\end{array}$ & $\begin{array}{c}-248.864^{* * *} \\
(84.869)\end{array}$ & $\begin{array}{c}216.224^{* * *} \\
(20.448)\end{array}$ & $\begin{array}{c}228.773 * * * \\
(44.435)\end{array}$ & $\begin{array}{c}-176.200^{* *} \\
(76.188)\end{array}$ \\
\hline d14 & $\begin{array}{c}-32.284^{* * *} \\
(12.508)\end{array}$ & $\begin{array}{c}-347.456^{* * *} \\
(68,061)\end{array}$ & $\begin{array}{c}-208.802^{* * * *} \\
(55.419)\end{array}$ & $\begin{array}{c}-109.681^{* * *} \\
(30.546)\end{array}$ & $\begin{array}{c}34.355^{* * *} \\
(7.704)\end{array}$ & $\begin{array}{c}80.470^{* * *} \\
(16,039)\end{array}$ & $\begin{array}{c}-28.289 \\
(27.559)\end{array}$ \\
\hline $\mathrm{d} 14 * \mathrm{~F}$ & $\begin{array}{l}-19.704 \\
(41.324)\end{array}$ & $\begin{array}{l}-420.706^{*} \\
(222.185)\end{array}$ & $\begin{array}{c}132.371 \\
(187.299)\end{array}$ & $\begin{array}{c}-102.881 \\
(103.264)\end{array}$ & $\begin{array}{l}-16.579 \\
(24.966)\end{array}$ & $\begin{array}{c}-129.992^{* * *} \\
(54.192)\end{array}$ & $\begin{array}{c}-154.253^{*} \\
(92.912)\end{array}$ \\
\hline I & $\begin{array}{c}338.281^{* * *} \\
(8.318)\end{array}$ & $\begin{array}{c}2,502.572^{* * *} \\
(46.425)\end{array}$ & $\begin{array}{c}522.523 * * * \\
(38.567)\end{array}$ & $\begin{array}{c}1,113.791^{* * *} * \\
(20.370)\end{array}$ & $\begin{array}{c}179.026^{* * *} \\
(5.112)\end{array}$ & $\begin{array}{c}590.633 * * * \\
(10.606)\end{array}$ & $\begin{array}{c}955.721^{* * *} \\
(18.195)\end{array}$ \\
\hline $\mathrm{E}$ & $\begin{array}{c}-5.653^{* * *} \\
(0.400)\end{array}$ & $\begin{array}{c}-33.571 * * * \\
(2.215)\end{array}$ & $\begin{array}{c}-4.760^{* * * *} \\
(1.816)\end{array}$ & $\begin{array}{c}-18.744 * * * * \\
(0.990)\end{array}$ & $\begin{array}{c}4.405^{* * *} \\
(0.245)\end{array}$ & $\begin{array}{c}14.103^{* * *} \\
(0.512)\end{array}$ & $\begin{array}{c}9.429 * * * \\
(0.878)\end{array}$ \\
\hline D & $\begin{array}{l}-3.868 \\
(2.777)\end{array}$ & $\begin{array}{c}255.915^{* * *} \\
(14.905)\end{array}$ & $\begin{array}{c}-50.191^{* * *} \\
(12.511)\end{array}$ & $\begin{array}{c}63.562^{* * * *} \\
(6.793)\end{array}$ & $\begin{array}{c}20.418^{* * *} \\
(1.700)\end{array}$ & $\begin{array}{c}63.882^{* * * *} \\
(3.576)\end{array}$ & $\begin{array}{c}103.260^{* * * *} \\
(6.149)\end{array}$ \\
\hline $\mathrm{T}$ & $\begin{array}{c}22.547^{* * * *} \\
(3.119)\end{array}$ & $\begin{array}{c}-298.570 * * * \\
(17.338)\end{array}$ & $\begin{array}{c}-99.904^{* * *} \\
(14.547)\end{array}$ & $\begin{array}{c}135.677^{* * *} \\
(7.622)\end{array}$ & $\begin{array}{c}2.151 \\
(1.925)\end{array}$ & $\begin{array}{c}55.041^{* * *} \\
(4.011)\end{array}$ & $\begin{array}{c}-38.765^{* * * *} \\
(6.889)\end{array}$ \\
\hline C & $\begin{array}{c}-2,733.008 * * * \\
(73.922)\end{array}$ & $\begin{array}{c}-24,452.289 * * * \\
(416.443)\end{array}$ & $\begin{array}{c}-7,576.820^{* * * *} \\
(366.519)\end{array}$ & $\begin{array}{c}-10,279.314^{* * * *} \\
(180.724)\end{array}$ & $\begin{array}{c}-2,071.026^{* * *} \\
(45.895)\end{array}$ & $\begin{array}{c}-6,198.796 * * * \\
(94.372)\end{array}$ & $\begin{array}{c}-9,354.548^{* * *} \\
(161.500)\end{array}$ \\
\hline $\mathrm{n}$ & 28,479 & 28,479 & 28,479 & 28,479 & 28,479 & 28,479 & 28,479 \\
\hline
\end{tabular}

Errores estándar en paréntesis

$* * * \mathrm{p}<0.01,{ }^{* *} \mathrm{p}<0.05,{ }^{*} \mathrm{p}<0.1$

\begin{tabular}{|c|c|c|c|c|c|c|c|}
\hline VARS & $\begin{array}{c}(8) \\
\text { Utensilios } \\
\end{array}$ & $\begin{array}{c}(9) \\
\text { Enseres }\end{array}$ & $\begin{array}{l}(10) \\
\text { Salud } \\
\end{array}$ & $\begin{array}{c}\text { (11) } \\
\text { Atención médica }\end{array}$ & $\begin{array}{c}\text { (12) } \\
\text { Hospitalización }\end{array}$ & $\begin{array}{c}\text { (13) } \\
\text { Transporte público }\end{array}$ & $\begin{array}{c}\text { (14) } \\
\text { Transporte foránce }\end{array}$ \\
\hline $\mathrm{F}$ & $\begin{array}{c}-211.867 * * * \\
(56.225)\end{array}$ & $\begin{array}{c}-8.465 \\
(106.974)\end{array}$ & $\begin{array}{c}-923.996 * * * \\
(190.807)\end{array}$ & $\begin{array}{c}-794.855^{* * *} \\
(209.269)\end{array}$ & $\begin{array}{c}-3,301.299 * * * \\
(1,123.790)\end{array}$ & $\begin{array}{c}-675.240^{* * *} \\
(99.410)\end{array}$ & $\begin{array}{c}-402.810^{* * *} \\
(133.142)\end{array}$ \\
\hline $\mathrm{d} 14$ & $\begin{array}{c}29.320 \\
(19.808)\end{array}$ & $\begin{array}{c}8.460 \\
(40.745)\end{array}$ & $\begin{array}{c}146.115^{* *} \\
(66.302)\end{array}$ & $\begin{array}{l}155.474^{* *} \\
(72.689)\end{array}$ & $\begin{array}{c}-250.721 \\
(347.257)\end{array}$ & $\begin{array}{l}215.494 * * * \\
(33.902)\end{array}$ & $\begin{array}{l}-169.668 * * * \\
(47.287)\end{array}$ \\
\hline $\mathrm{d} 14 * \mathrm{~F}$ & $\begin{array}{c}-118.934^{*} \\
(69.228)\end{array}$ & $\begin{array}{l}-60.366 \\
(130.280)\end{array}$ & $\begin{array}{l}-200.629 \\
(232.472)\end{array}$ & $\begin{array}{c}-551.673^{* *} \\
(257.022)\end{array}$ & $\begin{array}{c}1,189.066 \\
(1,340.208)\end{array}$ & $\begin{array}{c}-91.233 \\
(120.548)\end{array}$ & $\begin{array}{c}0.191 \\
(164.670)\end{array}$ \\
\hline I & $\begin{array}{l}328.871^{* * * *} \\
(13.309)\end{array}$ & $\begin{array}{l}881.336 * * * \\
(27.996)\end{array}$ & $\begin{array}{c}1,092.333 * * * \\
(43.922)\end{array}$ & $\begin{array}{c}1,112.123^{* * * *} \\
(48.276)\end{array}$ & $\begin{array}{c}2,695.658^{* * *} \\
(243.196)\end{array}$ & $\begin{array}{c}439.414 * * * \\
(22.839)\end{array}$ & $\begin{array}{l}656.255^{* * *} \\
(31.412)\end{array}$ \\
\hline $\mathrm{E}$ & $\begin{array}{l}-6.105^{* * *} \\
(0.642)\end{array}$ & $\begin{array}{l}-19.154^{* * *} \\
(1.329)\end{array}$ & $\begin{array}{c}10.062^{* * *} \\
(2.115)\end{array}$ & $\begin{array}{c}12.424^{* * * *} \\
(2.319)\end{array}$ & $\begin{array}{c}-118.006^{* * *} \\
(12.673)\end{array}$ & $\begin{array}{l}-9.197^{* * *} \\
(1.095)\end{array}$ & $\begin{array}{l}7.171^{* * *} \\
(1.528)\end{array}$ \\
\hline D & $\begin{array}{l}-4.388 \\
(4.397)\end{array}$ & $\begin{array}{l}-5.485 \\
(8.881)\end{array}$ & $\begin{array}{l}31.835 * * \\
(14.693)\end{array}$ & $\begin{array}{c}21.853 \\
(16.036)\end{array}$ & $\begin{array}{l}113.034 \\
(77.411)\end{array}$ & $\begin{array}{c}-16.700 * * \\
(7.577)\end{array}$ & $\begin{array}{l}76.735^{* * *} \\
(10.464)\end{array}$ \\
\hline $\mathrm{T}$ & $\begin{array}{c}3.931 \\
(4.953)\end{array}$ & $\begin{array}{c}-38.985^{* * *} \\
(10.238)\end{array}$ & $\begin{array}{l}29.343^{*} \\
(16.556)\end{array}$ & $\begin{array}{c}58.349 * * * \\
(18.089)\end{array}$ & $\begin{array}{c}550.735 * * * \\
(84.715)\end{array}$ & $\begin{array}{c}220.058^{* * * *} \\
(8.450)\end{array}$ & $\begin{array}{c}-84.238 * * * \\
(12.067)\end{array}$ \\
\hline C & $\begin{array}{c}-3,842.592^{* * *} \\
(119.447)\end{array}$ & $\begin{array}{c}-9,648.061^{* * *} \\
(254.179)\end{array}$ & $\begin{array}{c}-13,136.918^{* * *} \\
(392.957)\end{array}$ & $\begin{array}{c}-14,783.146^{* * *} \\
(436.094)\end{array}$ & $\begin{array}{c}-43,885.918^{* * *} \\
(2,342.188)\end{array}$ & $\begin{array}{c}-4,527.223^{* * *} \\
(203.795)\end{array}$ & $\begin{array}{c}-9,375.987^{* * * *} \\
(287.140)\end{array}$ \\
\hline $\mathrm{n}$ & 28,479 & 28,479 & 28,479 & 28,479 & 28,479 & 28,479 & 28,479 \\
\hline
\end{tabular}

Errores estándar en paréntesis

${ }^{* * *} \mathrm{p}<0.01,{ }^{* *} \mathrm{p}<0.05,{ }^{*} \mathrm{p}<0.1$ 
Impacto de la homologación del IVA en el consumo de los hogares... • Rolando Israel Valdez Ramírez y Emilio Hernández Gómez

\begin{tabular}{|c|c|c|c|c|c|c|c|}
\hline VARS & $\begin{array}{c}\text { (15) } \\
\text { Adquisición de vehículo }\end{array}$ & $\begin{array}{c}(16) \\
\text { Mantenimiento de vehículo }\end{array}$ & $\begin{array}{c}(17) \\
\text { Refacciones } \\
\end{array}$ & $\begin{array}{c}\text { (18) } \\
\text { Combustible } \\
\end{array}$ & $\begin{array}{c}(19) \\
\text { Comunicación }\end{array}$ & $\begin{array}{c}(20) \\
\text { Educación }\end{array}$ & $\begin{array}{c}\text { (21) } \\
\text { Esparcimiento }\end{array}$ \\
\hline F & $\begin{array}{c}4,095.258 \\
(2,972.774)\end{array}$ & $\begin{array}{l}1,331.305^{* * *} \\
(147.994)\end{array}$ & $\begin{array}{l}64.065 \\
(87.303)\end{array}$ & $\begin{array}{l}1,331.387^{* * *} \\
(138.258)\end{array}$ & $\begin{array}{c}161.360 * * * \\
(53.938)\end{array}$ & $\begin{array}{c}-2,531.689 * * * \\
(436.941)\end{array}$ & $\begin{array}{c}5.607 \\
(98.820)\end{array}$ \\
\hline $\mathrm{d} 14$ & $\begin{array}{c}311.283 \\
(1,269.914)\end{array}$ & $\begin{array}{c}76.276 \\
(59.287)\end{array}$ & $\begin{array}{l}-16.140 \\
(35.269)\end{array}$ & $\begin{array}{l}94.261^{*} \\
(55.590)\end{array}$ & $\begin{array}{l}-157.130^{* * *} \\
(20.028)\end{array}$ & $\begin{array}{c}98.111 \\
(152.269)\end{array}$ & $\begin{array}{l}-132.583^{* * *} \\
(37.887)\end{array}$ \\
\hline $\mathrm{d} 14^{*} \mathrm{~F}$ & $\begin{array}{c}-4,457.173 \\
(3,710.157)\end{array}$ & $\begin{array}{l}-888.155^{* * *} \\
(182.116)\end{array}$ & $\begin{array}{c}-86.566 \\
(107.403)\end{array}$ & $\begin{array}{l}-869.528^{* * *} \\
(170.248)\end{array}$ & $\begin{array}{l}-271.236^{* * *} \\
(66.042)\end{array}$ & $\begin{array}{l}-160.674 \\
(531.396)\end{array}$ & $\begin{array}{c}-246.388^{* *} \\
(121.397)\end{array}$ \\
\hline I & $\begin{array}{l}16,263.288^{* * *} \\
(913.103)\end{array}$ & $\begin{array}{l}2,865.743 * * * \\
\quad(41.005)\end{array}$ & $\begin{array}{l}939.201 * * * \\
(25.135)\end{array}$ & $\begin{array}{l}2,610.094^{* * * *} \\
(38.502)\end{array}$ & $\begin{array}{l}1,139.685^{* * * *} \\
(13.629)\end{array}$ & $\begin{array}{l}3,116.982^{* * *} \\
(103.108)\end{array}$ & $\begin{array}{c}1,666.769 * * * \\
(26.172)\end{array}$ \\
\hline $\mathrm{E}$ & $\begin{array}{c}-391.694^{* * *} \\
(44.477)\end{array}$ & $\begin{array}{l}10.899^{* * *} \\
(1.922)\end{array}$ & $\begin{array}{l}-1.238 \\
(1.155)\end{array}$ & $\begin{array}{c}10.511^{* * *} \\
(1.802)\end{array}$ & $\begin{array}{c}7.106^{* * *} \\
(0.643)\end{array}$ & $\begin{array}{c}-112.858^{* * *} \\
(5.276)\end{array}$ & $\begin{array}{c}-10.118 * * * \\
(1.222)\end{array}$ \\
\hline D & $\begin{array}{l}-479.716^{*} \\
(273.025)\end{array}$ & $\begin{array}{c}311.815^{* * *} \\
(12.751)\end{array}$ & $\begin{array}{l}75.719 * * * \\
(7.502)\end{array}$ & $\begin{array}{l}293.864^{* * *} \\
(11.951)\end{array}$ & $\begin{array}{c}119.130^{* * *} \\
(4.409)\end{array}$ & $\begin{array}{c}667.005^{* * *} \\
(33.828)\end{array}$ & $\begin{array}{c}127.738^{* * * *} \\
(8.242)\end{array}$ \\
\hline $\mathrm{T}$ & $\begin{array}{l}523.902^{*} \\
(306.266)\end{array}$ & $\begin{array}{c}22.206 \\
(14.680)\end{array}$ & $\begin{array}{c}-38.275 * * * \\
(8.861)\end{array}$ & $\begin{array}{c}37.180^{* * * *} \\
(13.755)\end{array}$ & $\begin{array}{c}0.498 \\
(5.015)\end{array}$ & $\begin{array}{c}1,923.001^{* * * *} \\
(39.926)\end{array}$ & $\begin{array}{c}-102.617^{* * *} \\
(9.542)\end{array}$ \\
\hline C & $\begin{array}{c}-217,310.377^{* * *} \\
(9,060.606)\end{array}$ & $\begin{array}{c}-32,077.583^{* * *} \\
(380.637)\end{array}$ & $\begin{array}{c}-11,537.744^{* * *} \\
(241.261)\end{array}$ & $\begin{array}{c}-29,517.222^{* * *} \\
(358.414)\end{array}$ & $\begin{array}{c}-11,693.049 * * * \\
(122.763)\end{array}$ & $\begin{array}{c}-40,435.709^{* * *} \\
(922.864)\end{array}$ & $\begin{array}{c}-17,122.108^{* * * 1} \\
\quad(237.424)\end{array}$ \\
\hline $\mathrm{n}$ & 28,479 & 28,479 & 28,479 & 28,479 & 28,479 & 28,479 & 28,479 \\
\hline
\end{tabular}

Errores estándar en paréntesis

$* * * \mathrm{p}<0.01, * * \mathrm{p}<0.05, * \mathrm{p}<0.1$

\begin{tabular}{|c|c|c|c|c|}
\hline VARS & $\begin{array}{c}(22) \\
\text { Paquetes turisticos }\end{array}$ & $\begin{array}{c}\text { (23) } \\
\text { Cuidado personal }\end{array}$ & $\begin{array}{c}(24) \\
\text { Accesorios personales }\end{array}$ & $\begin{array}{c}(25) \\
\text { Otros gastos }\end{array}$ \\
\hline $\mathrm{F}$ & $\begin{array}{c}-2,264.448 * * * \\
(658.672)\end{array}$ & $\begin{array}{c}138.038^{* * * *} \\
(42.179)\end{array}$ & $\begin{array}{l}-55.683 \\
(37.488)\end{array}$ & $\begin{array}{c}-2,186.067^{* * *} \\
(263.323)\end{array}$ \\
\hline $\mathrm{d} 14$ & -318.883 & $-102.151^{* * *}$ & $-24.795^{*}$ & $-147.089^{*}$ \\
\hline $\mathrm{d} 14^{*} \mathrm{~F}$ & $\begin{array}{c}-1,327.502 \\
(848.305)\end{array}$ & $\begin{array}{l}-54.681 \\
(51.433)\end{array}$ & $\begin{array}{l}-88.659^{*} \\
(46.579)\end{array}$ & $\begin{array}{c}-53.7 .931 \\
(330.060)\end{array}$ \\
\hline I & $\begin{array}{c}4,044.339 * * * \\
(171.434)\end{array}$ & $\begin{array}{l}646.386^{* * * *} \\
(10.080)\end{array}$ & $\begin{array}{c}273.551^{* * * *} \\
(9.523)\end{array}$ & $\begin{array}{c}1,856.198^{* * * *} \\
(57.519)\end{array}$ \\
\hline $\mathrm{E}$ & $\begin{array}{c}-46.616^{* * * *} \\
(8.018)\end{array}$ & $\begin{array}{c}-2.740 * * * \\
(0.486)\end{array}$ & $\begin{array}{c}-3.887 * * * \\
(0.457)\end{array}$ & $\begin{array}{l}28.529 * * * \\
(2.759)\end{array}$ \\
\hline D & $\begin{array}{c}318.670^{* * * *} \\
(51.290)\end{array}$ & $\begin{array}{c}51.761^{* * *} \\
(3.404)\end{array}$ & $\begin{array}{c}6.932^{* *} \\
(3.067)\end{array}$ & $\begin{array}{c}235.753^{* * *} \\
(18.504)\end{array}$ \\
\hline $\mathrm{T}$ & $\begin{array}{l}-65.829 \\
(60.552)\end{array}$ & $\begin{array}{c}93.206^{* * * *} \\
(3.814)\end{array}$ & $\begin{array}{c}-13.115^{* * *} \\
(3.523)\end{array}$ & $\begin{array}{c}-91.306^{* * *} \\
(21.512)\end{array}$ \\
\hline $\mathrm{C}$ & $\begin{array}{c}-53,882.686^{* * *} \\
(1,704.744)\end{array}$ & $\begin{array}{c}-5,774.021 * * * \\
(89.490)\end{array}$ & $\begin{array}{c}-3,252.053^{* * * *} \\
(86.883)\end{array}$ & $\begin{array}{c}-24,941.272^{* * *} \\
(536.882)\end{array}$ \\
\hline $\mathrm{n}$ & 28,479 & 28,479 & 28,479 & 28,479 \\
\hline
\end{tabular}

Errores estándar en naréntesis 
Números anteriores:

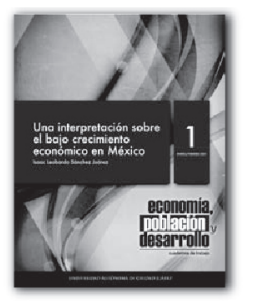

Economía, población y desarrollo.
Cuadernos de trabajo №1

Enero-Febrero 2011
Una interpretación sobre el bajo

crecimiento economico en México
Isaac Leobardo Sánchez Juárez

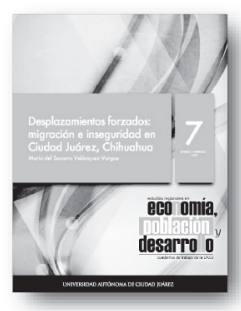

Economia, población y desarroll

Cuadernos de trabajo $\mathrm{N}$ -
Encro-Fcbrcro 2012

Desplazamientos forzados
migración e inseguridad

Ciudad Juárez, Chihuahua
Maria del Socorro Velázquez Varga

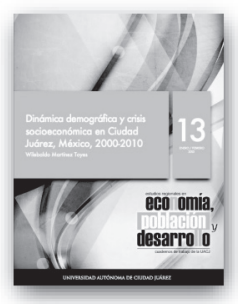

Economiá, población y desarrollo

de trabajo $N$

Dinámica demográ́fica y crisis
socieconómica en Ciudad Juáre Mexico, 2000-2010

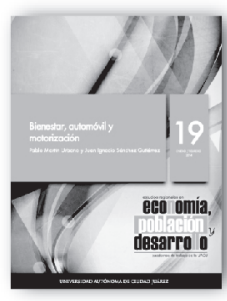

Economía, población y desarroll Enero - Febrero 2014 Bienestar, automóvil y motorización
Pablo Martín Urbano y Miosánchez Gutiérrez

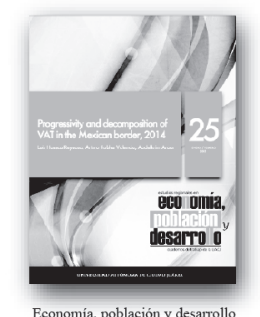

Cuadernos de trabajo No $_{0}$
Conomia,

Encro - Febrero 2015

Progressivity and decomposition of
VAT in the Mexican border, 2014 Abdelkim Araar

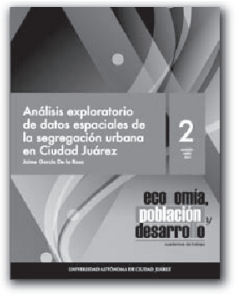

Economía, población y desarrollo.
Cuadernos de trabajo № 2

Marzo-Abril 2011

espaciales de la segregacón

Jaime Garcia De la Rosa

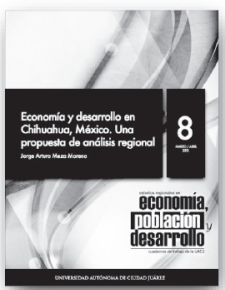

Economia, población y desarrollo

Cuadernos de trabajo №7

Economía y desarrollo en

propuesta de análisis regiona

Jorge Arturo Meza Moreno

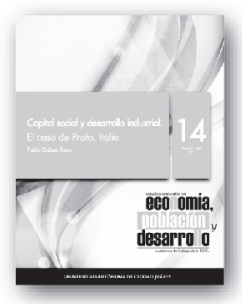

Economía, población y desarrollo

Marzo - Abri1 2013

Capital social y desarrollo

Pablo Galaso Reca

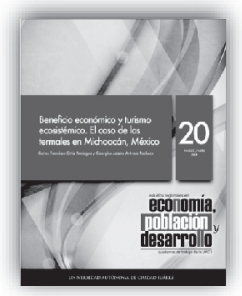

Economia, población y desarrollo

Marzo - Abril 2014

Beneficio económico y turismo

enico. El caso de las ter
en Michoacán, Mésico

arlos Franciseo Ortiz Paniagu

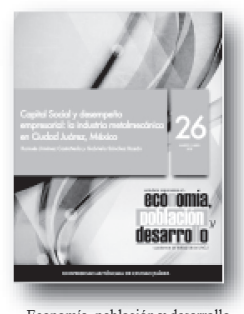

Economía, población y desarrollo

Cuadernos de trabajo № 26
Marzo - Abril 2015

Capital Social y desempeño empresa
la industria metalmecánica en

a industria metalmecánica en
Ciudad Juárez, México

Ramsés Jiménez Castañeda y
Gabriela Sáncez Bazán
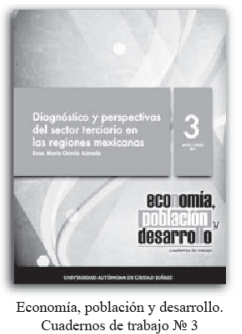

Diagnóstico y perspectivivas

regiones mexicanas
rosa Mária Garcia Almad

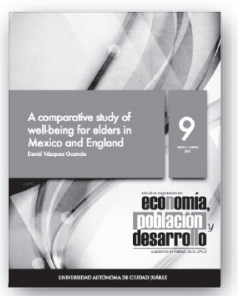

Economia, población y desarrollo

Cuadernos de trabajo № 9
Mayo - Junio 2012

A comparative study of

Mexico and England
David Vázquez Guzmán

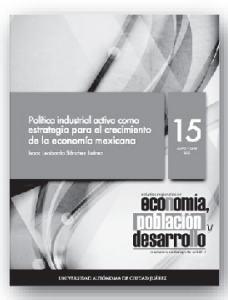

Economia, población y desarroll

Madernos de trabajo
Mayo - Junio 2013

Politica ind ustrial activa como

strategia para el crecimiento

Isaac Lcobardo Ś́nexicana
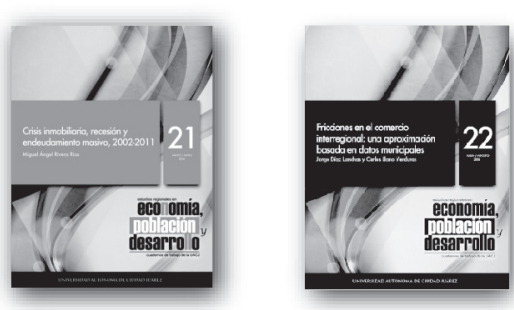

Economia, población y desarrollo

Cuademos de trabajo
Mayo - Junio 2014

Crisis inmobiliaria, recesión y
adeudamiento masivo, $2002-2011$

Miguel Ángel Rivera Rios

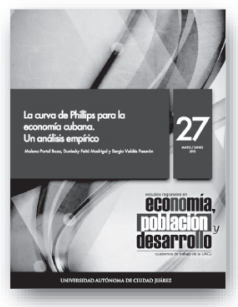

Economia, población y desarrollo

Cuadernos de trabajo № 27

a curva de Phillips para

economía cubana.

Malena Portal Boza, Duniesky Feitó

Economía, población y desarrollo.
Cuadernos de trabsio

julio-Agosto 201

Los indices IDH y FGT en la

mera década del siglo XX

Economía, población y decarrollo

Cuadernos de trabajo №
Julio - Agosto 2012

Political competition and the

edistribution in a federation

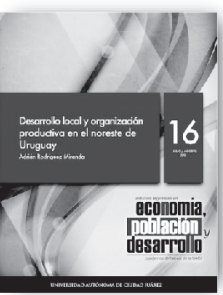

Economía, población y desarrollo

Cuadernos de trabajo №
Julio - Agosto 2013

Desarrollo local y organización
productiva en el noroeste de Urugu

Economía, población y desarrollo

Julio - Agosto 2014

Ficciones en el comercio

basada en datos municipales
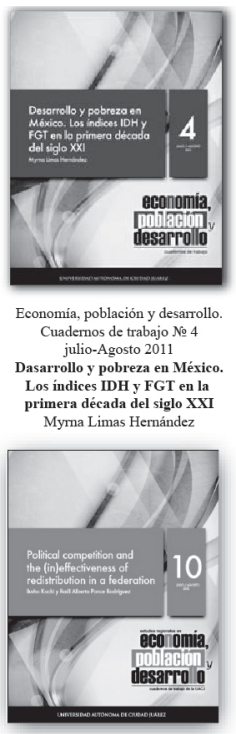

Jorge Diaz Lanchas y Carlos Llano Verduras
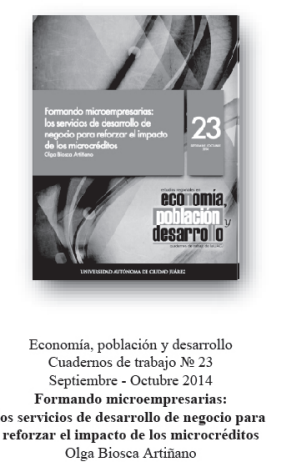

Cuadernos de trabajo № 24

El crecimiento de las regiones

el paradigma del desarrollo

divergente. Un marco térico
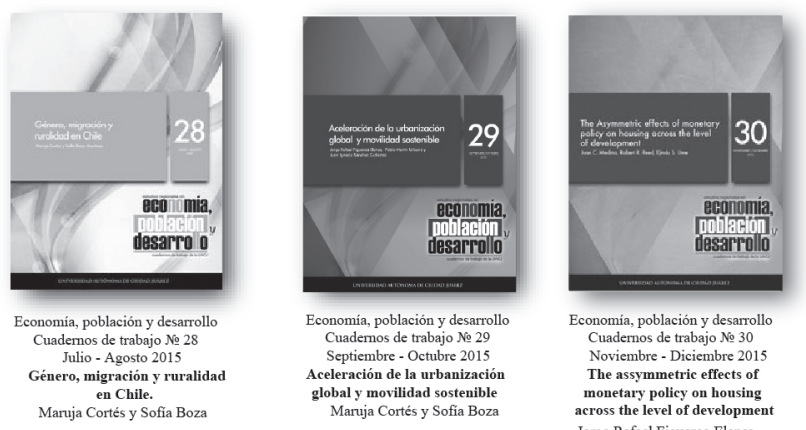

conomía, población y desarrol

Cuadernos de trabajo № 30

The assymmetric effects of

monetary policy on housing

Jorge Rafael Figueroa Elenes,

Juan Ignacio Sánchez Gutiérrez 
Números anteriores:
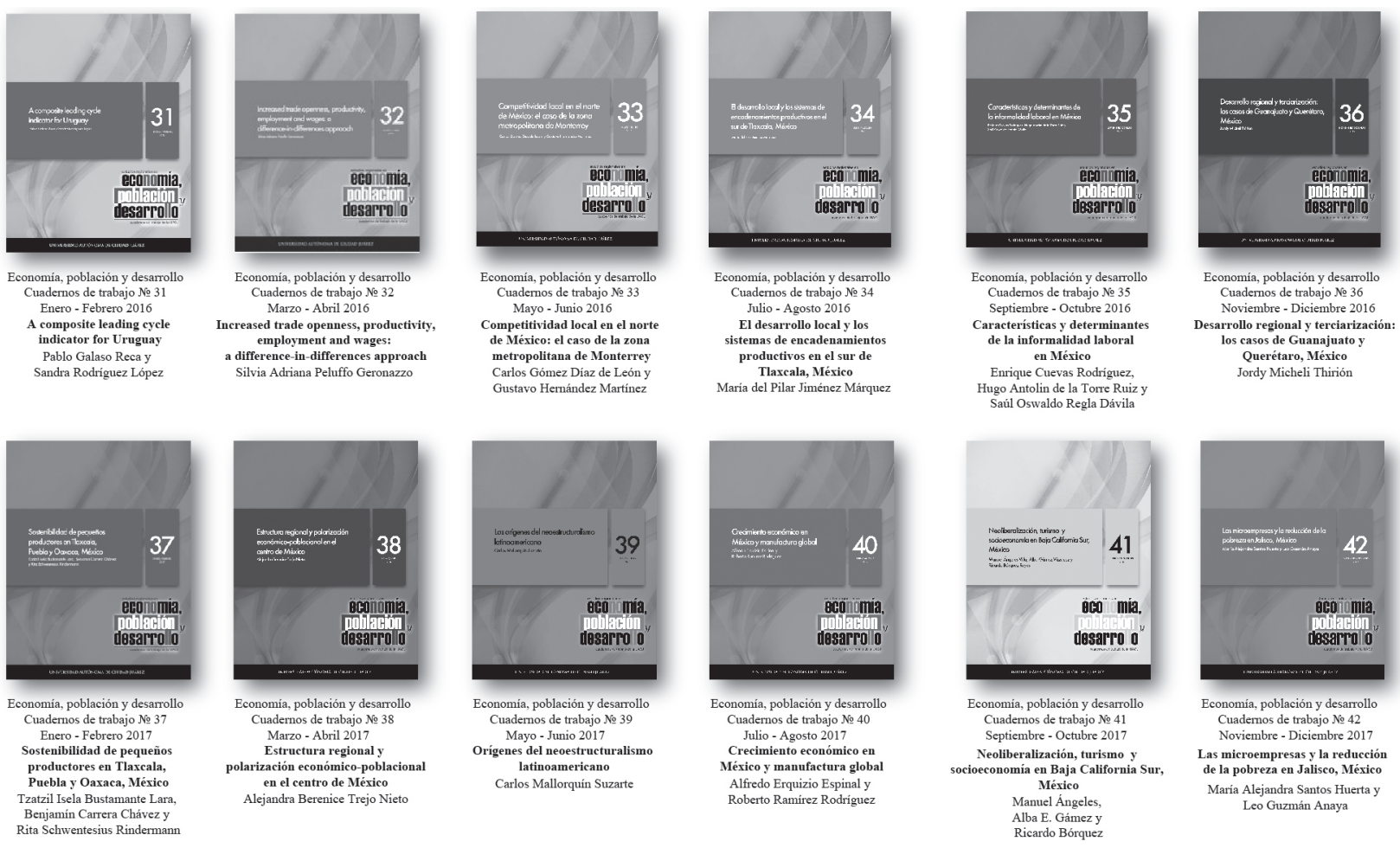

Cuadernos de trabajo № 42 Noviembre - Diciembre 2017 Las microempresas y la reducción
de la pobreza en Jallisco, México Maria Alcjandra Santos Hucrta y Alcjandra Santos Huc
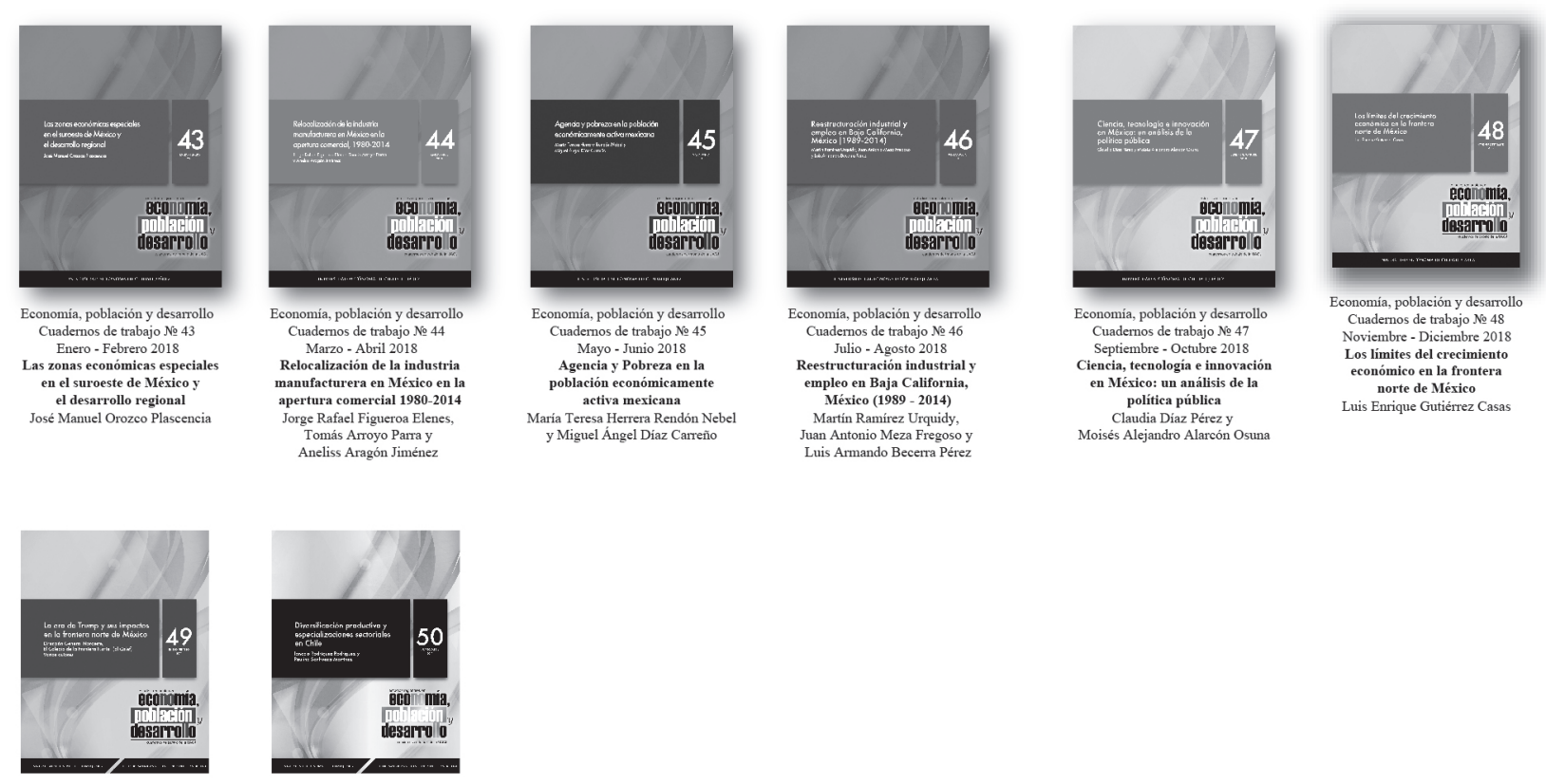

Economía, población y desarroll
Cuadernos de trabajo № 49 Cuadernos de trabajo № 40
Enero - Febrero 2019 Enero - Febrero 2019
La era de Trump y sus impactos
en la frontera norte de Mésico Dirección General de Meste Varios autores

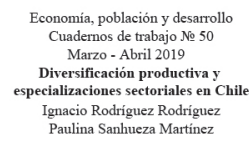




\section{$\rightarrow$ Normas Editoriales}

\section{Para el documento general:}

Tipo de letra: Times New Roman.

Tamaño: 11 puntos.

Interlineado: 1.5 espacios.

Títulos y subtítulos:

El texto principal en 11 puntos. Títulos 12 puntos (en resaltado). Subtítulos 11 puntos. Cada título y subtítulo deberá numerarse bajo el siguiente orden: $1,1.1,2,2.1,2.2 \ldots$

La extensión máxima de los cuadernos de trabajo será de 40 cuartillas.

La primera vez que se emplee una sigla en el texto se especificará primero su equivalencia completa y después la sigla.

\section{Hoja de presentación:}

Título:

14 puntos, centrado, resaltado.

Nombre de autor(es):

12 puntos

Resumen y abstract:

Debe incluir resumen en español y abstract (diez puntos), no mayor a 250 palabras

Palabras clave:

Incluir entre tres y cinco palabras clave, en español e inglés

Referencia del autor o autores:

Institución de adscripción, grado académico y líneas-grupos de investigación que desarrolla y a los que pertenece.

\section{Sistema de referencia de citas:}

Harvard-APA

Las citas bibliográficas en el texto deberán incluir entre paréntesis sólo el apellido del autor, la fecha de publicación y el número de página; por ejemplo: (Quilodrán, 2001: 33).

\section{Notación en sección de bibliografía y fuentes de información:}

Se deberá incluir al final del texto. Toda referencia deberá estar mencionada en el texto o notas de pie de página.

Cada referencia iniciará con el primer apellido o los apellidos, luego el nombre del autor, y después, entre paréntesis, el año de publicación seguido de un punto. Ejemplos:

Se deberá incluir al final del texto. Toda referencia deberá estar mencionada en el texto o notas de pie de página.

Cada referencia iniciará con el primer apellido o los apellidos, luego el nombre del autor, y después, entre paréntesis, el año de publicación seguido de un punto. Ejemplos:

Artículo:

Ros, Jaime (2008). “La desaceleración del crecimiento económico en México desde 1982”, en Trimestre Económico, vol. 75, núm. 299, pp. 537-560.

Libro:

Villarreal, René (2005). Industrialización, competitividad y desequilibrio externo en México. 
Un enfoque macroindustrial y financiero (1929-2010), México, Fondo de Cultura Económica. Capítulo de libro:

Castillo, Manuel Ángel (2003). "La política de inmigración en México: un breve recuento", en Manuel Ángel Castillo, Alfredo Lattes y Jorge Santibáñez (coords.), Migración y fronteras, Tijuana, El Colegio de la Frontera Norte / Asociación Latinoamericana de Sociología / El Colegio de México, pp. 425-451.

\section{Notas de pie de página:}

Se utilizarán para hacer indicaciones complementarias, aclaraciones o ampliación de una explicación. La notas de pie de página en Times New Roman, 10 puntos.

\section{Tipología de imágenes dentro del texto:}

Cuadro

Gráfica

Diagrama

Mapa

Figura

Todas las imágenes deben ser numeradas y mencionadas dentro del texto. A toda imagen debe incluirse la fuente.

Las indicaciones de la imagen: tipo y número de imagen, título de imagen y fuente se escriben en 10 puntos. En el texto poner como imagen los mapas, figuras, gráficas y diagramas -con el ánimo de no perder el formato realizado por el autor.

\section{Ecuaciones y fórmulas:}

Si se utilizan ecuaciones o fórmulas deberá utilizarse el editor de ecuaciones de Word y numerarse.

\section{Envío de trabajos}

Los trabajos deben ser enviados a la dirección de correo: lgtz@uacj.mx. Con el Dr. Luis Enrique Gutierrez Casas, editor de esta publicación.

La aceptación de cada colaboración dependerá de la evaluación de dos dictaminadores especialistas en la materia que se conservarán en el anonimato, al igual que el autor (autores) para efectos de la misma. 


\section{$\rightarrow$ Editorial Guidelines}

\section{For General Document:}

Font type: Times New Roman.

Size: font size 11 .

Paragraph: 1.5 line spacing.

Titles and subtitles: Main text font size 11. Titles font size 12 (Bold). Subtitles font size 11.

Each title and subtitle should be numbered in the following order: 1, 1.1, 2, 2.1, 2.2...

The maximum length of the workbooks will be 40 pages.

The first time an abbreviation is used in the text will be specified first complete equivalence and then stands.

\section{Front cover:}

Title:

Font size 14, centered, Bold.

Author name(s):

Font size 12.

Abstract:

It should include abstract in Spanish and abstract (font size 10), no more than 250 words.

Keywords:

Include three to five keywords, in Spanish and English.

Reference of author:

Institution of affiliation, academic degree and line-developed by research groups and belonging.

\section{Bibliographical appointment system:}

Harvard-APA

Citations in the text should include between parentheses only the author's name, publication date and page number, for example:

(Quilodrán, 2001: 33).

\section{Notation about Bibliography section and Information fonts:}

Should be included at the end of the text. All references must be mentioned in the text or footnotes page.

Each reference starts with the first name or last name, then the name of the author, and then, in parentheses, the year of publication followed by a period. Examples:

Article:

Ros, Jaime (2008). "La desaceleración del crecimiento económico en México desde 1982”, en Trimestre Económico, vol. 75, núm. 299, pp. 537-560.

Book:

Villarreal, René (2005). Industrialización, competitividad y desequilibrio externo en México. Un enfoque macroindustrial y financiero (1929-2010), México, Fondo de Cultura Económica.

Book chapter:

Castillo, Manuel Ángel (2003). "La política de inmigración en México: un breve recuento”, en Manuel Ángel Castillo, Alfredo Lattes y Jorge Santibáñez (coords.), Migración y fronteras, Tijuana, El Colegio de la Frontera Norte / Asociación Latinoamericana de Sociología / El Colegio de México, pp. 425-451. 


\section{Footnotes:}

Must be used to make additional indications, clarification or expansion of an explanation. The footnotes must be in Times New Roman, font size 10.

\section{Image typology inside text:}

Picture

Graph

Diagram

Map

Figure

All images must be numbered and mentioned in the text, should include the source image. The indications of the image: type and number of image, image title and source are written in 10 font size. In the text set as image maps, figures, graphs and charts-with the intention of not losing the formatting by the author.

\section{Equations and Formulae:}

When using equations or formulas should be used in Microsoft Word equation editor and numbered.

\section{Paper sending}

Entries must be sent to the email address: lgtz@uacj.mx. With Dr. Luis Enrique Gutiérrez Casas, editor of this publication.

Acceptance of each collaboration will depend on the evaluation of two examiners skilled in the art to be kept anonymous, like the author(s) for the same purposes. 
Esta obra se editó y terminó de imprimir en Ciudad Juárez, Chihuahua, México 
Cuadernos de Trabajo de la Universidad Autónoma de Ciudad Juárez, número 51, mayo - junio de 2019

\section{Director y editor}

Dr. Luis Enrique Gutiérrez Casas

\section{Comité editorial}

\section{Sección internacional}

Dra. Sofía Boza Martínez (Universidad de Chile, Chile)

Dra. Olga Biosca Artiñano (Glasgow Caledonian University, Reino Unido) Dra. Ángeles Sánchez Díez (Universidad Autónoma de Madrid, España)

Dr. Thomas Fullerton Mankin (University of Texas at El Paso, Estados Unidos)

Dr. Adrián Rodríguez Miranda (Universidad de la República, Uruguay)

Dra. Ikuho Kochi (Kanazawa University, Japón)

\section{Sección local}

(Universidad Autónoma de Ciudad Juárez)

Dra. Myrna Limas Hernández Dra. Rosa María García Almada Dr. Raúl Alberto Ponce Rodríguez Dr. Isaac Leobardo Sánchez Juárez Dr. Héctor Alonso Barajas Bustillos Dr. Juan Carlos Medina Guirado

Universidad Autónoma de Ciudad Juárez Cuadernos de Estudios Regionales en Economía, Población y Desarrollo ISSN 2007-3739 Cuerpo Académico de Estudios Regionales en Economía, Población y Desarrollo

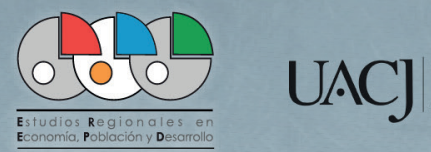

CUERPOS ACADÉMICOS

www.estudiosregionales.mx

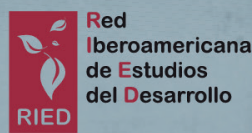

Publicación afiliada a la Red Iberoamericana de Estudios del Desarrollo

(c) Universidad Autónoma de Ciudad Juárez Avenida Plutarco Elías Calles \#1210, Fovissste Chamizal Ciudad Juárez, Chih., México. www.uacj.mx 